\title{
Correlação da força de preensão palmar e estado nutricional em idosas praticantes de atividade física
}

Correlation of the palmar pressure strength and nutritional state in elderly practitioners of physical activity

Correlación de la resistencia a la presión palmar y el estado nutricional en practicantes mayores de la actividad física

\author{
Ana Letícia Silva Nascimento Costa \\ ORCID: https://orcid.org/0000-0002-9734-914X \\ Faculdade Pitágoras Bacabal, Brasil \\ E-mail: analeticia.lety@hotmail.com \\ Raysa dos Santos Rego \\ ORCID: https://orcid.org/0000-0003-3917-4422 \\ Faculdade Pitágoras Bacabal, Brasil \\ E-mail: raysadossantos12@gmail.com \\ Adriana Miranda Sousa \\ ORCID: https://orcid.org/ 0000-0002-1290-7246 \\ Faculdade Pitágoras Bacabal, Brasil \\ E-mail: adrianamirandasousa@hotmail.com \\ Ruan Guilherme de Oliveira França \\ ORCID: https://orcid.org/0000-0002-2870-8401 \\ Universidade Federal do Piauí, Brasil \\ E-mail: ruanguilhermefranca@gmail.com \\ Bruna Cruz Magalhães \\ ORCID: https://orcid.org/0000-0002-1491-8694 \\ Faculdade Pitágoras Bacabal, Brasil \\ E-mail: brunacruzmagalhaes@gmail.com \\ Camila Guedes Borges de Araujo \\ ORCID: https://orcid.org/0000-0001-5403-5258 \\ Faculdade Pitágoras Bacabal, Brasil \\ E-mail: camilaguedes2@gmail.com
}

\begin{abstract}
Resumo
O presente estudo teve como objetivo avaliar a força de preensão palmar e correlação com o estado nutricional de mulheres idosas. Estudo com abordagem transversal, quantitativo com amostra composta por 60 idosas com idade > 60 anos do sexo feminino, realizado no Centro de Convivência Dos Idosos, localizado em uma cidade do Nordeste Brasileiro. Foram estudadas varáveis para avaliação da composição corporal. O consumo alimentar foi avaliado por meio de Questionário de Frequência Alimentar (QFA) adaptado para idosos. Os dados da Força de Preensão Palmar (FPP) foram avaliados por meio da aplicação de formula. Para diferenças significativas adotou-se $\mathrm{p}<0,05$. A pesquisa

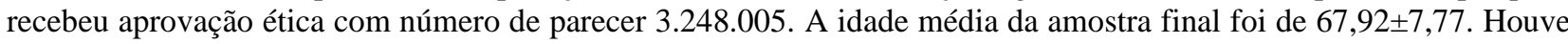
prevalência de sobrepeso (45\%) segundo o IMC. A ingestão calórica esteve acima da recomendação $(1907,38 \pm 783,07)$. Entre as participantes com idades entre 60 a 69 anos de idade as médias tanto de FPP-D (26,70 + $2,37)$ como FPP-ND $(25,23+1,73)$ apresentaram-se adequadas, já entre as que possuíam idade a cima de 70 anos a média da FFP-ND $(18,93+3,55)$ esteve abaixo do esperado, encontrando-se adequado apenas o valor de FPP-D $(20,53+3,73)$. Os resultados obtidos neste estudo sugerem que a força muscular das idosas se mantém preservada, o que influencia no desempenho funcional dessa população. O estado nutricional inadequado com sobrepeso das idosas deste estudo, é resultante do consumo de alimentos com densidade calórica elevada e baixa qualidade de macro e micronutrientes.
\end{abstract}

Palavras-chave: Envelhecimento; Idosas; Força muscular; Estado nutricional.

\footnotetext{
Abstract

The present study aimed to assess handgrip strength, nutritional status and its relationship with functional limitations in the elderly. A cross-sectional, quantitative study with a sample of 60 elderly women> 60 years old, carried out at the Centro de Convivência Dos Idosos, located in the city of Northeast Brazilian. Variables were studied to assess body composition. Food consumption was assessed using the Food Frequency Questionnaire (FFQ) adapted for the
} 
elderly. The Palmar Grip Force (FPP) data were evaluated using the formula. For significant differences, $\mathrm{p}<0.05$ was adopted. The research received ethical approval with opinion number 3,248,005. The mean age of the final sample was $67.92 \pm 7.77$. There was a prevalence of overweight $(45 \%)$ according to BMI. Caloric intake was above the recommendation $(1907.38 \pm 783.07)$. Participants aged 60 to 69 years old, the means for both FPP-D $(26.70+2.37)$ and FPP-ND $(25.23+1.73)$ were adequate, among those who aged over 70 years, the mean FFP-ND $(18.93+3.55)$ was below expectations, with only the FPP-D value $(20.53+3.73)$ being adequate. The results obtained in this study suggest that the muscle strength of the elderly is preserved, which influences the functional performance of this population. The inadequate nutritional status of overweight women in this study is the result of consuming foods with high caloric density and low quality of macro and micronutrients.

Keywords: Aging; Elderly; Muscular strength; Nutritional status.

\section{Resumen}

El presente estudio tuvo como objetivo evaluar la fuerza de prensión, el estado nutricional y su relación con las limitaciones funcionales en los ancianos. Estudio transversal, cuantitativo, con una muestra de 60 ancianas> 60 años, realizado en el Centro de Convivência Dos Idosos, ubicado en la ciudad del Nordeste de Brasil. Se estudiaron variables para evaluar la composición corporal. El consumo de alimentos se evaluó mediante el Cuestionario de frecuencia alimentaria (FFQ) adaptado para personas mayores. Los datos de Palmar Grip Force (FPP) se evaluaron mediante la fórmula. Para diferencias significativas, se adoptó una p <0.05. La investigación recibió aprobación ética con el número de opinión 3.248.005. La edad media de la muestra final fue 67,92 $\pm 7,77$. Hubo una prevalencia de sobrepeso (45\%) según IMC. La ingesta calórica estuvo por encima de la recomendación (1907,38 $\pm 783,07)$. Participantes de 60 a 69 años, las medias para FPP-D $(26,70+2,37)$ y FPP-ND $(25,23+1,73)$ fueron adecuadas, entre los mayores de 70 años, la media de FFP-ND $(18,93+3,55)$ estuvo por debajo de las expectativas, siendo adecuado solo el valor FPP-D $(20,53+3,73)$. Los resultados obtenidos en este estudio sugieren que se conserva la fuerza muscular de los ancianos, lo que influye en el desempeño funcional de esta población. El inadecuado estado nutricional de las mujeres con sobrepeso en este estudio es el resultado del consumo de alimentos con alta densidad calórica y baja calidad de macro y micronutrientes.

Palabras clave: Envejecimiento; Ancianos; Fuerza muscular; Estado nutricional.

\section{Introdução}

De acordo com a Lei 10.741/2003 é considerado idoso o indivíduo com 60 anos ou mais; e atualmente pode-se observar que a população idosa no Brasil obteve um aumento na prevalência e incidência destes (Valença et al., 2017). Em pesquisa realizada pelo Centro de Políticas Sociais da Fundação Getulio Vargas (FGV Social) com dados da Organização Mundial da Saúde (OMS), constatou-se aumento de 20\% no número de pessoas com idades acima de 65 anos entre os anos de 2012 a 2018. Estima-se que a população com idades acima de 60 anos corresponda a 28 milhões de pessoas, representando $13 \%$ da população total. Considerando dados mundiais, observa-se associação do número de idosos a condição socioeconômica do país, o Brasil ocupa colocação intermediária no ranking, assumindo a posição de $31^{\circ}$ entre os $20 \%$ mais ricos, e $80^{\circ}$ entre os $20 \%$ mais pobres (Neri et al., 2020).

Durante o processo de envelhecimento, ocorrem várias modificações, tais como diminuição da massa corporal magra, aumento da quantidade de tecido gorduroso, declínio da estatura e peso, perda de músculos esqueléticos, diminuição da força e da massa muscular. A presença de má nutrição, doenças crônicas não transmissíveis (DCNT) e a inatividade física afetam ainda mais essas alterações, intensificando as limitações funcionais em idosos (Guedes et al., 2016; Ponti et al., 2020).

Nesse sentido, sabe-se que a Força de Preensão Palmar (FPP) é importante na prática de atividades do cotidiano, como por exemplo, realizar trabalhos domésticos, segurar algum objeto, atividade de autocuidado, enfim, manter a autonomia das atividades diárias de um indivíduo (Wagner et al., 2014). Com o avanço da idade, o decremento da força muscular é comum, e esta interfere no equilíbrio do indivíduo e consequentemente na sua limitação funcional (Bohannon, 2019).

Desse modo, é considerável que não se pode dispensar o fato da utilização de um dinamômetro para se obter a força de preensão palmar, entretanto pela escassez deste instrumento, Novaes et al. (2009) elaborou equações para predizer valores normativos para FPP, além desse, Moura (2008) determinou um ponto de referência de FPP por ausência de um ponto de corte exato (Soares et al., 2019).

A força muscular atinge seu ponto mais alto por volta dos 30 anos de idade e se mantem satisfatoriamente conservado 
até os 50 anos (Wagner et al., 2019). A partir deste, ocorre perda da força muscular, sendo incomoda diminuição após os 60 anos, e evidente em idosos entre 70 e 80 anos, na qual verifica-se o declínio da força muscular em um percentual que varia de 20 a 40\% (Franchi et al., 2019; Ganapathy; Nieves 2020).

Outra vertente a se destacar na saúde do idoso é o estado nutricional, uma vez que as alterações deste com o envelhecimento estão relacionadas com as modificações corporais, além de estar associada com a limitação funcional e a incapacidade de realização das atividades de vida diárias (AVD), como também influência sobre o bem-estar físico e psicológico durante o processo de envelhecimento (Danielewicz et al., 2014; Silva et al., 2015; Venturini et al., 2015).

Dessa forma o consumo alimentar dos idosos, é essencial na manutenção do estado nutricional devido a diversos fatores, como ambiente social, alterações fisiológicas nas sensações gustativas, alterações na digestão e absorção de nutrientes e dificuldades funcionais para aquisição ou preparo dos alimentos (Gomes et al., 2016). Conforme Coelho-Jínior, (2018) e Martinez et al. (2016) os autores demostram que existe uma baixa ingestão de proteínas de alto valor biológico em idosos, visto que, o consumo inadequado de proteína está relacionado com a redução da massa muscular, que, por sua vez, associa-se à incapacidade física, pior qualidade de vida e maior mortalidade.

É compreensível que a população de idosos possui dificuldades de autonomia, devido modificações psicológicas, fisiológicas e morfológicas, dificultando até mesmo o estado nutricional dos idosos (Yiallouris et al., 2019). Considerando-se a carência de estudos que avaliam de forma precisa a força de preensão palmar, estado nutricional e sua relação com a limitação funcional na população de idosos; o seguinte estudo tem como intuito relacionar a limitação funcional do idoso e sua força de preensão palmar.

Além disso, compreender se o estado nutricional do idoso influencia na força de preensão palmar e investigar a existência de relação do processo de envelhecimento e a diminuição do consumo de proteína com a força muscular. Consequente a isso, o estudo pretende subsidiar dados para que, futuramente, englobem estratégias que possam auxiliar no estado nutricional adequado, força muscular, limitação funcional e consumo alimentar de idosos, e proporcionar possíveis melhorias na qualidade de vida dessa população. Diante desse contexto, o presente estudo teve como objetivo avaliar a força de preensão palmar e a correlação com o estado nutricional de mulheres idosas.

\section{Metodologia}

Trata-se de um estudo com abordagem transversal, quantitativo realizado no Centro de Convivência Dos Idosos, localizado na Estrada da Bela Vista s/n, cidade de Bacabal, MA. A amostra foi composta por idosas com idade $\geq 60$ anos do sexo feminino, cadastradas e participantes das atividades desenvolvidas no Centro de convivência Social, totalizando 60 idosas aptas e que atendiam aos critérios de inclusão e exclusão.

Para inclusão dos participantes foram considerados idosas com idade $\geq 60$ anos, praticantes de atividade física (dança e natação) por pelo menos 2 vezes/ semana e que aceitaram a participar da pesquisa e assinaram o Termo de Consentimento Livre e Esclarecido. Não foram incluídas idosas que apresentaram osteoartrite/osteoartrose de mão; doença de Parkinson; diagnóstico ortopédico, como amputação; portadores de problemas cognitivo; indivíduos com incapacidade de compreensão e comunicação e indivíduos do sexo masculino, pois estes podem modificar os resultados obtidos.

Foram avaliados a força de preensão palmar (FPP), peso, altura, circunferência da panturrilha (CP), circunferência do braço (CB), circunferência muscular do braço (CMB), prega cutânea tricipital (PCT), e aplicado o questionário de frequência alimentar (QFA).

Para obter os dados da FPP, se utilizou a fórmula de Novaes et al. (2009), para o sexo feminino da mão dominante e não dominante, e os valores médios adequados de FPP de Moura (2008), sendo 20,55 kgf para a FPP-D e 19,03 para a FPPND. 


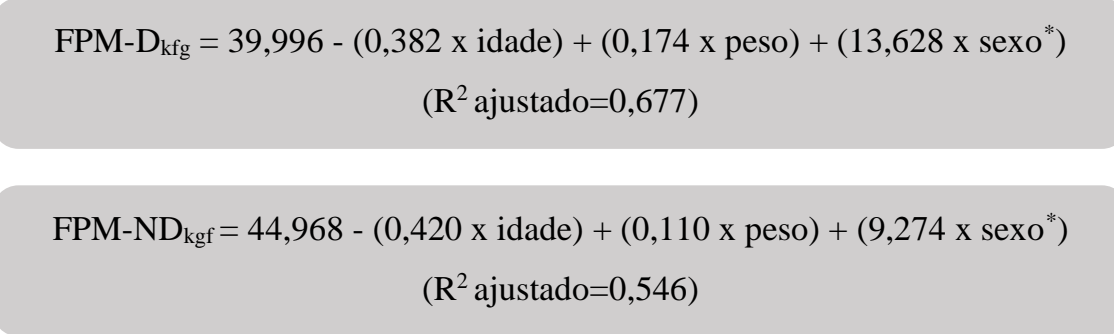

*Sexo: homem $=1$, mulher $=0$

O peso foi verificado através de uma balança digital da marca Mallory, com medida máxima de $150 \mathrm{~kg}$. Os indivíduos foram orientados a ficarem em posição ereta, com roupas leves e descalços, os pés juntos e os braços estendidos ao longo do corpo. A altura foi verificada com o estadiômetro portátil, na qual o idoso, se manteve em posição ereta, descalço, com os calcanhares juntos, costas retas e os braços estendidos ao longo do corpo (Chaves, 2013; Lustosa et al., 2016).

O IMC foi calculado a partir da razão entre o peso $(\mathrm{kg})$ e a altura $(\mathrm{m})$ ao quadrado. Para classificação do estado nutricional pelo IMC foram utilizados os pontos de corte propostos por Lipschitz (1994) para o grupo de idosos, sendo < 22 $\mathrm{kg} / \mathrm{m}^{2}$ desnutrição, 22 a $27 \mathrm{~kg} / \mathrm{m}^{2}$ eutrofia e $>27 \mathrm{~kg} / \mathrm{m}^{2}$ sobrepeso.

A circunferência da panturrilha e do braço foram mensurados através de uma fita métrica inelástica. Para obtenção dos dados da CP, o idoso ficou na posição sentado, com a perna formando um ângulo de $90^{\circ}$, onde a fita foi colocada na região mais protuberante da panturrilha, cujo o ponto adequado é maior ou igual a $31 \mathrm{~cm}$. Para a mensuração da $\mathrm{CB}$, o indivíduo foi colocado na posição supina, na qual foi retirado o ponto médio entre o acrômio e olecrano e em seguida o braço foi estendido de forma relaxado ao longo do corpo e medido (Cavalcante et al., 2017). A CMB foi obtida a partir dos valores da CB e PCT, por meio da equação (Valente et al., 2016):

\section{$\mathrm{CMB}(\mathrm{cm})=\mathrm{CB}(\mathrm{cm})-\pi[\mathrm{PCT}(\mathrm{mm} / 10)]$}

O adipômetro da marca Sanny, foi utilizado para a aferição da PCT. O examinador segurou firmemente a prega entre o polegar e o indicador da mão um centímetro acima da média da $\mathrm{CB}$, em seguida com a mão direita, o instrumento foi posicionado e suas extremidades fixadas no local marcado, permanecendo a pinçada por 4 segundos e repetindo esse procedimento três vezes, sendo os valores registrados imediatamente, para obtenção da média destes (da Paz et al., 2012). Foram usados os pontos de corte de Jelliffe (1996), para a CB, CMB e PCT para o sexo feminino, sendo $28,3 \mathrm{~cm}, 23,2 \mathrm{~cm}$ e $16,5 \mathrm{~mm}$, respectivamente.

Para a avaliação do consumo alimentar utilizou-se o QFA adaptado para idosos, visto que a diversas abordagens das diferentes populações, o QFA precisa ser adaptado para cada grupo etário, considerando uma série de questões relacionadas aos hábitos alimentares dos indivíduos específicos (Silva; Vasconcelos, 2012). Para análise quantitativa do QFA utilizou-se o software Dietsys versão 4.01 .

Os dados foram organizados em planilhas do Excel® (2010) para realização de análise descritiva das variáveis observadas nos grupos estudados. Posteriormente, os dados foram exportados para o programa SPSS (for Windows® versão 18.0) para análise estatística dos resultados. Inicialmente, avaliou-se a simetria da distribuição dos dados pelo teste de Kolmogorov-Smirnov, e a homogeneidade das variâncias pelo teste de Levene. O teste t independente e o teste Mann-Whitney foram utilizados para variável contínua paramétrica (força de preensão palmar dominante) e não paramétrica (força de preensão palmar não dominante), entre os grupos, respectivamente. O coeficiente de correlação linear de Pearson foi realizado 
para a análise das correlações. A diferença foi considerada estatisticamente significativa quando o valor de $\mathrm{p}<0,05$, adotandose um intervalo de confiança de $95 \%$.

O projeto foi submetido à aprovação prévia pela Comissão de Avaliação de Projetos de Pesquisa (CAPP/HU), e após aprovação foi submetido e aprovado pelo Comitê de Ética em Pesquisa do Centro Universitário do Maranhão (UNICEUMA), com Certificado de Apresentação e Apreciação Ética (CAAE) e parecer no 3.248.005. Todos os participantes assinaram o Termo de Consentimento Livre e Esclarecido (TCLE) e foram informados quanto à natureza da investigação, riscos e benefícios da pesquisa, conforme prediz a Resolução nº 466/2012, do Conselho Nacional de Saúde (Brasil, 2012).

\section{Resultados e Discussão}

A proposta da pesquisa foi avaliar o consumo alimentar e relacionar com o estado nutricional e a força de preensão palmar. O estudo demonstrou que as participantes estavam com sobrepeso, consumo calórico superior ao recomendado, FPP-D adequada e FPP-ND inadequada apenas para faixa etária de mulheres com idade superior a 70 anos.

A idade contribui para diminuição do metabolismo e hábitos alimentares inadequados, e o estado nutricional, nessa fase da vida, pode ser comprometido. No presente estudo pode-se observar que as idosas participantes apresentaram, de acordo com a média do índice de massa corporal (IMC) (Tabela 1), um nível elevado de $27,21 \mathrm{~kg} / \mathrm{m}^{2}$, sendo adequado para um indivíduo dessa idade $27 \mathrm{~kg} / \mathrm{m}^{2}$.

O idoso tende a diminuir seu peso, e dentre as principais consequências a esse fato, pode-se destacar a redução de tecido muscular, redução no peso das vísceras e perda de água corporal (Ponti et al., 2020). Todavia mulheres com até 75 anos, tendem a aumentar de peso (Kim, 2018), tornando claro que a média de $61,13 \mathrm{~kg}$ do total de peso das idosas induz no seu próprio IMC, visto que a média da idade deste estudo é de 67,92 anos (Tabela 1).

Sabe-se que o idoso passa por declínio da estatura com o avançar da idade, devido a redução dos discos intervertebrais, a redução da massa óssea, achatamento das vertebras dentre outras e que a diminuição da estatura de mulheres é de 1,5 cm por década (Mai et al., 2018; dos Santos et al., 2013). Verifica-se que a média da altura desse estudo foi de 1,50 m (Tabela 1), porém não se pode comprovar se houve diminuição da estatura dessas idosas. 
Tabela 1 - Média e desvio padrão de dados referentes às características gerais, composição corporal e contribuição energética de idosas.

\begin{tabular}{l|c|c}
\multicolumn{1}{c|}{ Parâmetros } & Média & DP \\
\hline Características Gerais & 67,92 & 7,77 \\
\hline Idade & 61,13 & 12,99 \\
\hline Peso (kg) & 1,50 & 0,06 \\
\hline Altura (m) & 27,21 & 5,52 \\
\hline IMC (kg/m ${ }^{2}$ ) & & \\
\hline Composição Corporal & & \\
\hline
\end{tabular}

Composição Corporal

\begin{tabular}{l|l|c}
\hline CB (cm) & 30,28 & 4,49 \\
\hline PCT (mm) & 26,07 & 4,91 \\
\hline CMB (cm) & 22,10 & 3,98 \\
\hline CP (cm) & 33,02 & 4,04 \\
\hline FPP-D (kgf) & 24,74 & 4,05 \\
\hline FPP-ND (kgf) & 23,23 & 3,82 \\
\hline
\end{tabular}

\section{Contribuição energética}

\begin{tabular}{l|c|c}
\hline Energia (kcal) & 1907,38 & 783,07 \\
\hline Carboidrato (\%) & 51,31 & 8,52 \\
\hline Proteína (\%) & 26,06 & 5,64 \\
\hline Lipídio (\%) & 27,59 & 5,65 \\
\hline Cálcio (mg) & 1600,58 & 1755,33 \\
\hline Vitamina D $(\boldsymbol{\mu g})$ & 12,84 & 14,52 \\
\hline
\end{tabular}

$\mathrm{DP}=$ desvio padrão; $\mathrm{CB}=$ circunferência do braço; $\mathrm{PCT}=$ prega cutânea tricipital; $\mathrm{CMB}=$ circunferência muscular do braço; $\mathrm{CP}=$ circunferência da panturrilha; FPP-D = força de preensão palmar dominante; FPP-ND = força de preensão palmar não dominante. Valores de referência: 1741,70 kcal/dia para o sexo feminino (IOM, 2005); 45 a 65\% de carboidratos, 10 a 35\% de proteínas e 20 a 35\% de lipídios;

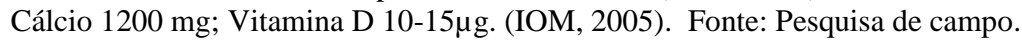

Tendo em vista as variáveis antropométricas, peso e altura identificou-se que $45 \%$ das idosas estão com sobrepeso, $40 \%$ eutróficas e $15 \%$ desnutridas, sendo necessários outros indicadores para determinar um estado nutricional confiável dessa população, pois o IMC não considera alterações que ocorrem durante o envelhecimento, implicando no desenvolvimento de estratégias para adequação do estado nutricional das idosas. Além disso, é importante salientar que valores superiores aos pontos de corte apresentam risco de desenvolvimento de doenças cardiovasculares e à medida que estes valores diminuem associa-se com doenças infecciosas e falta de apetite.

Lima et al. (2017) estudaram 170 idosos sendo 148 do gênero feminino e 22 do gênero masculino no Centro de Convivência para a Terceira idade no município de Vitória/ES, encontraram uma média em mulheres de $27,4 \mathrm{~kg} / \mathrm{m}^{2}$ com classificação do IMC sobrepeso, justificando em seu estudo que esta prevalência de excesso de peso pode estar relacionada com a inatividade física, alto consumo de alimentos calóricos decorrente de gordura saturada e rico em açúcar.

Pereira et al. (2016) em seu estudo realizado com os dados da Pesquisa de Orçamento Familiares (POF), 2008/2009, do Instituto Brasileiro de Geografia e Estatística (IBGE), totalizando 20.114 idosos, sendo 55,6\% do sexo feminino e 44,4\% do sexo masculino, obteve um IMC em mulheres com prevalência de 41,9\% sobrepeso, explicando em seu estudo que os valores elevados do IMC é predominante em idosos que moram sozinhos e dispõem de acesso sem restrição à alimentos inadequados, 
influenciando assim em seu estado nutricional. Além desse, é importante considerar os níveis de escolaridade e renda por serem fatores que aumentam a chance de escolha de alimentos com baixa qualidade nutricional.

Perante o processo de envelhecimento, a circunferência do braço (CB) sofre alterações pela diminuição da massa magra, pois esta abrange um conjunto constituído pelo tecido ósseo, epitelial, gorduroso e muscular. Destaca-se no presente estudo um valor médio superior ao de referência para o sexo feminino, segundo Jelliffe (1996), demonstrando adequação. No estudo realizado por Sass e Marcon (2015), com 429 idosos - 64,33\% do sexo feminino - residentes na área urbana de Sarandi/PR, notaram uma média de mulheres por idade de 32,99 (60-69), 32,03 (70-79) 28,79 ( $\geq 80$ ), e constataram que as médias da CB diminuem com o avançar da idade, evidenciando que a idade influencia na perda de massa magra.

Diante do exposto, a prega cutânea tricipital (PCT) é uma variável bastante utilizada em estudos com idosos, por ser um indicador de reserva calórica, que neste estudo demonstrou valor superior ao recomendado para o sexo feminino, caracterizando obesidade, segundo referência de Cuppari (2002). De acordo com os dados de estudo de Menezes et al. (2013), com número total de idosos entrevistados maior, de 806 (69,1\% mulheres), residentes habituais em domicílios particulares na zona urbana da cidade de Campina Grande/PB, apresentou-se valor médio significativamente superior $(22,0)$, propondo que mulheres contém maior reserva de gordura.

A circunferência muscular do braço (CMB) é um importante indicador de reserva de massa muscular, excluindo o tecido ósseo e adiposo (Bhattacharya et al., 2019; Deurenberg; Deurenberg-Yap, 2009). Em idosos a tendência durante o envelhecimento é que esta reserva diminua, devido as alterações sofridas por esta população. Diante disto, a média do estudo foi de 22,10 cm, valor inferior ao recomendado por Jelliffe (1996), porém, ainda se encontra adequado segundo Cuppari (2002). Este valor, com média próxima ao ponto de referência, deve-se pela média da idade ser constituída por idosas de 67,92 anos (considerado idoso jovem).

No estudo de Correia et al. (2014), com 316 idosos, totalizando 173 mulheres (54,7\%) e 143 homens encontraram valores da $\mathrm{CMB}$, menores em mulheres de 75 anos e mais, comparadas às mulheres de grupo etário de 60-64 anos, nesse as médias obtidas foram 22,65 (60-64), 21,54 (65-69), 21,46 (70-74), 21,45 (75-79) e 20,79 ( $\geq 80$ ). Esclarecendo que a redução da massa muscular e da gordura visceral é maior em mulheres e as alterações antropométricas são mais perspectiveis no grupo etário mais velho, supondo que a redução na massa muscular está associada à sarcopenia, e que esta pode influenciar na capacidade funcional e aumentar o risco de quedas.

Outro indicador da quantidade de massa muscular é a circunferência da panturrilha (CP), utilizada como um padrão que determina a desnutrição em idosos (Krzymińska-Siemaszko et al., 2020). Neste estudo encontrou-se uma média da CP de $33,02 \mathrm{~cm}$, considerada satisfatória, já que o adequado para idosos é igual ou superior a $31 \mathrm{~cm}$. Desse modo observa-se que a média da CP $(33,02 \mathrm{~cm}$ ), é devido ao estado nutricional do idoso (IMC 46\% de sobrepeso), assim apresentando forte correlação com a CP (r=0,810). No estudo de Cruz (2016), na qual foram entrevistadas $63 \%$ de idosas, verificaram que a CP apresentou massa magra preservada com 53,5\% das idosas e que o IMC $43,3 \%$ foi de baixo peso, 36,7\% eutróficas e $6,7 \%$ sobrepeso.

Tabela 2 - Médias e desvio padrão dos valores da FPP-D e FPP-ND em kgf.

\begin{tabular}{l|c|c|c}
\hline \multirow{4}{*}{ Parâmetros } & 60-69 ANOS & $>$ 70 ANOS & p \\
\cline { 2 - 4 } & Média \pm DP & Média \pm DP & \\
\hline FPP-D $^{\text {a }}$ & $26,70 \pm 2,37$ & $20,53 \pm 3,73$ & $<0,01$ \\
\hline FPP-ND $^{\mathbf{b}}$ & $25,23 \pm 1,73$ & $18,93 \pm 3,55$ & $<0,01$ \\
\hline
\end{tabular}

Valor de $\mathrm{p}<0,05$. ${ }^{\mathrm{a}}$ Teste t independente. ${ }^{\mathrm{b}}$ Mann-Whitney U. DP: desvio padrão. FPP-D: força de preensão palmar dominante. FPP-ND: força de preensão palmar não dominante. Fonte: Pesquisa de campo. 
Diante do exposto, no que diz respeito ao estado nutricional das mulheres idosas do estudo, a fim de contribuir com o estado nutricional e avaliar a realização de atividades domésticas diárias, um outro método, que faz parte da avaliação nutricional geriátrica é a força de preensão palmar (FPP), por ser um parâmetro que analisa a funcionalidade, a capacidade muscular e a desnutrição em idosos.

De acordo com as médias encontradas nesse estudo (Tabela 2) a partir da estratificação dos resultados por faixa etária, observou-se que entre as participantes com idades entre 60 a 69 anos de idade as médias tanto para FPP-D $(26,70 \pm 2,37)$ como para FPP-ND $(25,23 \pm 1,73)$ apresentaram-se adequadas segundo valores propostos por Moura (2008). Já para as idosas com idade acima de 70 anos de idade a média da FFP-ND $(18,93$ + 3,55) apresentou-se abaixo do esperado, encontrando-se adequado apenas o valor de FPP-D $(20,53 \pm 3,73)$. Esses dados, quando associados a estratificação da faixa etária, foram significativos e demonstraram diferença nas médias de FPP com valor superior nas idosas entre 60 e 69 anos de idade.

Outra vertente é o consumo de proteínas dessas idosas, que está dentro dos valores recomendados, sendo uma justificativa que se associa aos valores da CMB, CP e FPP. Quando se verifica a ingestão de proteína desse grupo no presente estudo de acordo com a gramatura por quilo de peso encontrou-se um resultado de $1,8 \mathrm{~g} / \mathrm{kg} / \mathrm{peso}$, sendo um valor considerado adequado em conformidade com as recomendações diária de proteína para idosos. Sabe-se que a dieta para idosos é fundamental para manter o aporte proteico adequado, pois a ingestão aumentada de proteína total, está relacionada com a menor perda de massa muscular (Franchi et al., 2019).

No entanto, a média de calorias totais consumidas pelas idosas foi de 1907,38 kcal (Tabela 1), valor superior ao recomendado da necessidade energética diária desses indivíduos (1741,70 kcal). Essa circunstância esclarece o IMC e a PCT, desse estudo, pois o consumo de alimentos inadequados com alto índice calórico prejudica o estado nutricional, ocasionando também alterações metabólicas.

Os resultados para a média de carboidratos e lipídios foram de 51,31 e 27,59 (Tabela 1), na qual estão em concordância com as recomendações diárias para esse grupo de faixa etária. Mas é necessário considerar que o consumo desses macronutrientes seja proveniente de alimentos com carboidratos simples e gorduras saturadas, explicando assim que as idosas estão acima do peso e massa magra reduzida, tendo em vista que esses alimentos de baixa qualidade nutricional são mais acessíveis quanto a praticidade e condição econômica.

Resultados semelhantes foram encontrados no estudo de Barazzetti et al. (2013), na qual a população estudada apresentou percentagem de proteína, carboidratos e lipídios adequados de acordo com as recomendações. Justificando em seu estudo que o elevado IMC, decorre da ingestão elevada de fontes de proteínas que apresentam gorduras como carnes, leites de gado/integral, entre outras. Valores similares foi evidenciado no estudo de da Silva Dórea et al. (2015), na qual as médias encontradas das idosas relacionadas a proteína, carboidrato e lipídios, apresentaram resultados adequado segundo a recomendação das DRIs.

Além do consumo adequado de macronutrientes, a vitamina $\mathrm{D}$ tem sido associada a massa e força muscular, visto que a mesma tem efeito protetor contra a degradação muscular, mesmo àquela induzida pelo envelhecimento, e no metabolismo do cálcio e dos ossos (Mieszkowski et al, 2018; Rafiq et al., 2017). Sua forma de atuação pode se dar pela preservação celular por meio da regulação positiva da folistatina, um inibidor endógeno da miostatina que, por sua vez, promove a degeneração muscular (Aspell et al., 2019; Remelli et al., 2019). Outro meio está na geração do ambiente anabólico promovido por este micronutriente, uma vez que estimula a produção de insulina e a atividade da leucina, participantes da sinalização da via Akt/mTOR, culminando em aumento da síntese proteica e, consequentemente, favorecendo a manutenção da massa magra em níveis satisfatórios (Dzik; Kaczor, 2019). 
O consumo desses micronutrientes, aliada a prática regular de atividade física influencia na funcionalidade da maioria dos sistemas do corpo humano. Diante disso, pode-se observar o consumo adequado por parte das mulheres participantes do estudo (Tabela 1).

A ingestão adequada de proteínas, também demonstrada neste estudo, aumenta o fator de crescimento semelhante à insulina 1 (IGF-1) e promove a absorção de cálcio, bem como o consumo de vitamina D contribui para essa absorção. Dessa forma, reduzem potencialmente a perda óssea, diminuem as quedas e fraturas (Hill et al., 2019). Apesar do consumo de proteína não está associado com a FPP, os resultados deste estudo demonstraram correlação de cálcio e vitamina D, apesar de fraca, com a FPP-D e -ND (Tabela 3).

Quando se observa a correlação da FPP-D (Tabela 3), verifica-se que esta possui correlação negativa com a idade, cálcio e vitamina $\mathrm{D}$, e positiva com IMC, $\mathrm{CB}, \mathrm{CMB}, \mathrm{CP}$, parâmetros que durante o estudo mostram-se com resultados que interferem na composição muscular dessas idosas, sendo que o IMC foi visto como inadequado, já a CMB está abaixo do proposto por Jelliffe (1996) e a PCT está acima do esperado.

Estas variações sustentam tanto a adequação da FPP-D encontrada entre as participantes com idades entre 60 a 69 anos de idade como a inadequação entres as com mais de 70 anos, a qual representa e confirma a redução de massa magra e de força muscular. A perda da massa muscular e da força tende a ocorrer de forma progressiva entre idosos, consequentemente acometendo mais drasticamente aqueles com idades mais avançadas e influenciar a modulação da composição corporal em favor do aumento no percentual de massa gorda e redução na massa magra (Larsson et al., 2019).

Tabela 3 - Correlação da FPP-D e FPP-ND com os dados antropométricos.

\begin{tabular}{lcc|cc}
\hline \multirow{2}{*}{ Parâmetros } & \multicolumn{2}{c|}{ FPP-D } & & \multicolumn{2}{c}{ FPP-ND } \\
\cline { 2 - 5 } & $\mathrm{r}^{*}$ & $\mathrm{p}$ & $\mathrm{r}^{*}$ & $\mathrm{p}$ \\
\hline Idade & $\mathbf{- 0 , 8 2 3}$ & $\mathbf{0 , 0 0 0}$ & $\mathbf{0 , 9 1 3}$ & $\mathbf{0 , 0 0 0}$ \\
IMC & $\mathbf{0 , 5 4 1}$ & $\mathbf{0 , 0 0 0}$ & $\mathbf{0 , 3 8 5}$ & $\mathbf{0 , 0 0 2}$ \\
CB & $\mathbf{0 , 4 9 7}$ & $\mathbf{0 , 0 0 0}$ & $\mathbf{0 , 3 7 0}$ & $\mathbf{0 , 0 0 4}$ \\
PCT & 0,122 & 0,352 & 0,045 & 0,731 \\
CMB & $\mathbf{0 , 4 8 9}$ & $\mathbf{0 , 0 0 0}$ & $\mathbf{0 , 3 8 1}$ & $\mathbf{0 , 0 0 3}$ \\
CP & $\mathbf{0 , 5 2 5}$ & $\mathbf{0 , 0 0 0}$ & $\mathbf{0 , 3 9 0}$ & $\mathbf{0 , 0 0 2}$ \\
Calorias & $-0,098$ & 0,457 & $-0,121$ & 0,356 \\
\%Proteína & 0,012 & 0,927 & 0,017 & 0,900 \\
\%Carboidratos & $-0,164$ & 0,210 & $-0,162$ & 0,217 \\
\%Lipídios & 0,123 & 0,350 & 0,125 & 0,342 \\
Cálcio & $\mathbf{- 0 , 2 6 1}$ & $\mathbf{0 , 0 4 4}$ & $\mathbf{- 0 , 2 8 4}$ & $\mathbf{0 , 0 2 8}$ \\
Vitamina D & $\mathbf{- 0 , 2 5 5}$ & $\mathbf{0 , 0 4 9}$ & $\mathbf{0 , 2 7 4}$ & $\mathbf{0 , 0 3 4}$ \\
\hline
\end{tabular}

*Correlação de Pearson. Valor de p <0,05. DP: desvio padrão. FPP-D: força de preensão palmar dominante. FPP-ND: força de preensão palmar não dominante. Fonte: Pesquisa de campo.

Ainda sobre a força muscular, as correlações entre essas medidas e a FPP-ND foram fracas (Tabela 3), e somente a idade manteve-se com correlação muito forte negativa quando comparada com FPP-D. Esse achado pode ser explicado, pela periodicidade da utilização da mão dominante em atividades de vida diárias que requerem maior esforço, contrapondo com a 
não dominante, que é utilizada em tarefas simples do cotidiano que requerem apenas o movimento dos pequenos músculos do nosso corpo.

Desta forma, faz-se necessário considerar a idade das participantes do estudo, uma vez que acima dos 80 anos de idade a diminuição da FPP torna-se mais evidente. É importante considerar também o seu nível de atividade física notoriamente ativo, dado que as mesmas são praticantes de zumba e natação no Centro de Convivência Social, sugerindo tanto a importância das modificações fisiológicos sofridas por esta população quanto a influência da prática de atividade física como fator protetor contra a perda da força e da massa muscular.

\section{Conclusão}

Os resultados obtidos neste estudo sugerem que a força muscular das idosas se mantém preservada, o que influencia no desempenho funcional desta população, possuindo assim autonomia em realizar suas atividades de vida diária. A manutenção dessa força é proveniente do alto consumo de proteína e um nível de atividade física regular. Vale ressaltar que os valores abaixo do esperado para a força de preensão palmar não dominante entre as participantes com idades acima de 70 anos pode estar associado as alterações fisiológicas implícitas ao envelhecimento.

O estado nutricional inadequado com sobrepeso das idosas deste estudo, é resultante do consumo de alimentos com índices elevados de calorias e baixa qualidade de macro e micronutrientes. Torna-se evidente que o acompanhamento nutricional é primordial para um bom estado nutricional, auxiliando diretamente nas alterações decorrente do envelhecimento, diminuindo o número de doenças crônicas, mortalidades e reduzindo gastos com a saúde.

Nesse contexto, o envelhecimento ativo deve ser incentivado, visto que essas idosas ainda estão em média de idade jovem, para que essas tenham durante o envelhecimento, autonomia, saúde e qualidade de vida. Portanto é necessário a realização de trabalhos futuros, no intuito de subsidiar programas e políticas de saúde para encorajar a participação de idosos em atividades sociais, de lazer e culturais.

\section{Referências}

Aspell, N., Laird, E., Healy, M., Lawlor, B., \& O'Sullivan, M. (2019). Vitamin D deficiency is associated with impaired muscle strength and physical performance in community-dwelling older adults: findings from The English Longitudinal Study Of Ageing. Clinical interventions in aging, $14,1751$.

Barazzetti, R., Siviero, J., \& Bonatto, S. (2013). Estado nutricional, consumo de calorias e macronutrientes de mulheres participantes de uma universidade da terceira idade no sul do país. Estudos Interdisciplinares sobre o Envelhecimento, 18(2).

Bhattacharya, A., Pal, B., Mukherjee, S., \& Roy, S. K. (2019). Assessment of nutritional status using anthropometric variables by multivariate analysis. BMC public health, 19(1), 1-9.

Bohannon, R. W. (2019). Grip strength: an indispensable biomarker for older adults. Clinical interventions in aging, $14,1681$.

Brasil. (2012). Resolução, N. 466/2012 do Conselho Nacional de Saúde. Diretrizes e normas.

Cavalcante, L. S., Coutinho, P. T. D. Q., \& Burgos, M. G. P. A. (2017). Aplicabilidade da MAN-Mini Avaliação Nutricional em Idosos diabéticos. Nutr Clin Diet Hosp, 37(1), 67-74.

Chaves, L. R., Sousa, C. M. M., Martins, M. C. C. et al. (2013). Estado nutricional e consumo alimentar de idosos assistidos na estratégia saúde da família. Journal of Nursing, 7(12), 6780-9.

Coelho-Júnior, H. J., Rodrigues, B., Uchida, M., \& Marzetti, E. (2018). Low protein intake is associated with frailty in older adults: A systematic review and meta-analysis of observational studies. Nutrients, 10(9), 1334.

Correia, A. R. B., Coqueiro, R. D. S., Santos, M. C., Leal Neto, J. D. S., Queiroz, B. M. D., Barbosa, A. R., \& Fernandes, M. H. (2014). Anthropometric reference values for community-dwelling older adults from northeastern Brazil. Revista Brasileira de Cineantropometria \& Desempenho Humano, 16(5), 494503.

Cruz, L. D. (2016). Avaliação antropométrica e percentual de gordura em idosos sem doença crônica não transmissível e não acamados, internados no hospital regional de Itabaiana. HU Revista, 42(3).

Cuppari, L. (2002). Guias de Medicina Ambulatorial e Hospitalar. Nutrição Clínica no Adulto: Manole. 
da Paz, R. D. C., Fazzio, D. M. G., \& dos Santos, A. L. B. (2012). Avaliação nutricional em idosos institucionalizados. Revista de Divulgação Científica Sena Aires, 1(1), 9-18.

da Silva Dórea, G., Manochio-Pina, M. G., \& dos Santos, D. (2015). Aspectos nutricionais de idosos praticantes de atividade física. DEMETRA: Alimentação, Nutrição \& Saúde, 10(2), 347-360.

Danielewicz, A. L., Barbosa, A. R., \& Del Duca, G. F. (2014). Nutritional status, physical performance and functional capacity in an elderly population in southern Brazil. Revista da Associação Médica Brasileira, 60(3), 242-248.

Deurenberg, P., \& Deurenberg-Yap, M. (2009). Ageing and changes in body composition: the importance of valid measurements. In Food for the ageing population (pp. 169-183). Woodhead Publishing.

dos Santos, R. R., Bicalho, M. A. C., Mota, P., de Oliveira, D. R., \& de Moraes, E. N. (2013). Obesidade em idosos. Rev Med Minas Gerais, $23(1)$, 64-73.

Dzik, K. P., \& Kaczor, J. J. (2019). Mechanisms of vitamin D on skeletal muscle function: oxidative stress, energy metabolism and anabolic state. European journal of applied physiology, 119(4), 825-839.

Franchi, M. V., Monti, E., Carter, A., Quinlan, J. I., Herrod, P. J., Reeves, N. D., \& Narici, M. V. (2019). Bouncing back! counteracting muscle aging with plyometric muscle loading. Frontiers in physiology, 10, 178.

Ganapathy, A., \& Nieves, J. W. (2020). Nutrition and Sarcopenia-What Do We Know?. Nutrients, 12(6), 1755.

Gomes, A. P., Soares, A. L. G., \& Gonçalves, H. (2016). Baixa qualidade da dieta de idosos: estudo de base populacional no sul do Brasil. Ciência \& Saúde Coletiva, 21, 3417-3428.

Gouveia, L. A. G. D., \& Oliveira, A. D. (2014). Nutritional risk and associated factors in elderly patients with congestive heart failure in a Brazilian cardiology hospital. Revista Brasileira de Geriatria e Gerontologia, 17(2), 265-274.

Guedes, J. M., Bortoluzzi, M. G., Matte, L. P., Andrade, C. M. D., Zulpo, N. C., Sebben, V., \& Tourinho Filho, H. (2016). Efectos Del Entrenamiento Combinado Sobre La Fuerza, Resistencia Y La Potencia Aeróbica En Mujeres Mayores. Revista Brasileira de Medicina do Esporte, $22(6), 480-484$.

Hill, T. R., Verlaan, S., Biesheuvel, E., Eastell, R., Bauer, J. M., Bautmans, I., Brandt, K., Donini, L. M., Maggio, M., Mets, T., Seal, C. J., Wijers, S. L., Sieber, C., Cederholm, T., Aspray, T. J., \& Provide Consortium (2019). A Vitamin D, Calcium and Leucine-Enriched Whey Protein Nutritional Supplement Improves Measures of Bone Health in Sarcopenic Non-Malnourished Older Adults: The Provide Study. Calcified tissue international, 105(4), 383-391

Institute of Medicine. (2005). Dietary reference intakes for energy, carbohydrate, fiber, fat, fatty acids, cholesterol, protein, and amino acids (Vol. 5, pp. 589768). National Academy Press: Washington, DC, USA.

Jelliffe, D. B., \& World Health Organization. (1966). The assessment of the nutritional status of the community (with special reference to field surveys in developing regions of the world. World Health Organization.

Kim, T. N. (2018). Elderly obesity: is it harmful or beneficial?. Journal of obesity \& metabolic syndrome, $27(2), 84$.

Krzymińska-Siemaszko, R., Deskur-Śmielecka, E., Kaluźniak-Szymanowska, A., Lewandowicz, M., \& Wieczorowska-Tobis, K. (2020). Comparison of diagnostic performance of SARC-F and its two modified versions (SARC-CalF and SARC-F+ EBM) in community-dwelling older adults from Poland. Clinical Interventions in Aging, 15, 583.

Larsson, L., Degens, H., Li, M., Salviati, L., Lee, Y. I., Thompson, W., \& Sandri, M. (2019). Sarcopenia: aging-related loss of muscle mass and function. Physiological reviews, 99(1), 427-511.

Lima, L. M., Souza, R. J. S., Cunha, M. R. H. D., Leopoldo, A. S., \& Leopoldo, A. P. L. (2017). Prevalência de Sobrepeso e Obesidade em Idosas do Centro de Convivência para a Terceira Idade de Vitória. ES. Rev. Bras. Ciência da Saúde, 21(2), 119-26.

Lipschitz, D. A. (1994). Screening for nutritional status in the elderly. Primary care, 21(1), 55-67.

Lustosa, M. F., Sá, C. M. A. T. D., Cavalcanti, A. C., Medeiros, R. A. B. D., Nova, L. P. V., \& Pedrosa, R. P. (2016). Perfis metabólico e nutricional como preditores da síndrome da apneia obstrutiva do sono. Revista de Nutrição, 29(5), 665-678.

Mai, X., Marshall, B., Hovey, K. M., Sperrazza, J., \& Wactawski-Wende, J. (2018). Risk factors for 5-year prospective height loss among postmenopausal women. Menopause (New York, NY), 25(8), 883.

Martinez, B. P., Ramos, I. R., Oliveira, Q. C. D., Santos, R. A. D., Marques, M. D., Forgiarini Júnior, L. A., \& Camelier, A. A. (2016). Existe associação entre massa e força muscular esquelética em idosos hospitalizados?. Revista Brasileira de Geriatria e Gerontologia, 19(2), $257-264$.

Menezes, T. N. D., Brito, M. T., Araújo, T. B. P. D., Silva, C. C. M., Nolasco, R. R. D. N., \& Fischer, M. A. T. S. (2013). Perfil antropométrico dos idosos residentes em Campina Grande-PB. Revista brasileira de geriatria e gerontologia, 16(1), 19-27.

Mieszkowski, J., Niespodziński, B., Kochanowicz, A., Gmiat, A., Prusik, K., Prusik, K., Kortas, J., Ziemann, E., \& Antosiewicz, J. (2018). The Effect of Nordic Walking Training Combined with Vitamin D Supplementation on Postural Control and Muscle Strength in Elderly People-A Randomized Controlled Trial. International journal of environmental research and public health, 15(9), 1951.

Moura, P. M. D. L. (2008). Estudo da força de preensão palmar em diferentes faixas etárias do desenvolvimento humano.

Neri, I., Guglielmo, A., Virdi, A., Gaspari, V., Starace, M., \& Piraccini, B. M. (2020). The red half-moon nail sign: a novel manifestation of coronavirus infection. Journal of the European Academy of Dermatology and Venereology, 34(11), e663-e665. 
Novaes, R. D., Miranda, A. S. D., Silva, J. D. O., Tavares, B. V. F., \& Dourado, V. Z. (2009). Equações de referência para a predição da força de preensão manual em brasileiros de meia idade e idosos. Fisioterapia e Pesquisa, 16(3), 217-222.

Pereira, I. F. D. S., Spyrides, M. H. C., \& Andrade, L. D. M. B. (2016). Estado nutricional de idosos no Brasil: uma abordagem multinível. Cadernos de Saúde Pública, 32, e00178814.

Ponti, F., Santoro, A., Mercatelli, D., Gasperini, C., Conte, M., Martucci, M., \& Bazzocchi, A. (2020). Aging and imaging assessment of body composition: from fat to facts. Frontiers in endocrinology, 10, 861.

Rafiq, R., Prins, H. J., Boersma, W. G., Daniels, J. M., den Heijer, M., Lips, P., \& de Jongh, R. T. (2017). Effects of daily vitamin D supplementation on respiratory muscle strength and physical performance in vitamin D-deficient COPD patients: a pilot trial. International journal of chronic obstructive pulmonary disease, 12, 2583-2592.

Remelli, F., Vitali, A., Zurlo, A., \& Volpato, S. (2019). Vitamin D deficiency and sarcopenia in older persons. Nutrients, 11(12), 2861.

Sass, A., \& Marcon, S. S. (2015). Comparação de medidas antropométricas de idosos residentes em área urbana no sul do Brasil, segundo sexo e faixa etária. Revista Brasileira de Geriatria e Gerontologia, 18(2), 361-372.

Silva, N. D. A., Pedraza, D. F., \& Menezes, T. N. D. (2015). Physical performance and its association with anthropometric and body composition variables in the elderly. Ciencia \& saude coletiva, 20,3723-3732.

Silva, T. D. A., \& Vasconcelos, S. M. L. (2012). Procedimentos metodológicos empregados em questionários de frequência alimentar elaborados no Brasil: uma revisão sistemática. Revista de Nutrição, 25(6), 785-797.

Soares, V. D. M., Fernandes, M. H., de Queiroz, B. M., Brito, T. A., da Silva Coqueiro, R., \& Carneiro, J. A. O. (2019). Análise comparativa entre dinamometria e equações antropométricas preditoras da força de preensão manual em idosos. Revista Brasileira de Ciência e Movimento, 27(1), 11-17.

Valença, T. D. C., Santos, W. D. S., Lima, P. V., Santana, E. D. S., \& Reis, L. A. D. (2017). Deficiência física na velhice: um estudo estrutural das representações sociais. Escola Anna Nery, 21(1).

Valente, K. P., Silva, N. M. F., Faioli, A. B., Barreto, M. A., Moraes, R. A. G. D., \& Guandalini, V. R. (2016). Espessura do músculo adutor do polegar na avaliação nutricional de pacientes cirúrgicos. Einstein (São Paulo), 14(1), 18-24.

Venturini, C. D., Engroff, P., Sgnaolin, V., Kik, R. M. E., Morrone, F. B., Silva Filho, I. G. D., \& Carli, G. A. D. (2015). Consumo de nutrientes em idosos residentes em Porto Alegre (RS), Brasil: um estudo de base populacional. Ciência \& Saúde Coletiva, 20, $3701-3711$.

Wagner, J., Knaier, R., Infanger, D., Arbeev, K., Briel, M., Dieterle, T., ... \& Schmidt-Trucksäss, A. (2019). Functional aging in health and heart failure: The Complete Study. BMC cardiovascular disorders, 19(1), 1-17.

Wagner, P. R., Ascenço, S., \& Wibelinger, L. M. (2014). Hand grip strength in the elderly with upper limbs pain. Revista Dor, 15(3), $182-185$.

Yiallouris, A., Tsioutis, C., Agapidaki, E., Zafeiri, M., Agouridis, A. P., Ntourakis, D., \& Johnson, E. O. (2019). Adrenal aging and its implications on stress responsiveness in humans. Frontiers in endocrinology, 10, 54. 